\title{
Non-United Fracture Scaphoid Surgery, Simple Step by Step Precise Technique
}

\author{
Safa Eldin Abaza* \\ Consultant Orthopedic Surgeon, Gulf Diagnostic Center Hospital, UAE
}

*Corresponding author : Safaa Eldin Abaza, Consultant Orthopedic Surgeon, Gulf Diagnostic Center Hospital- Abu Dhabi, UAE.

Received Date: February 25, 2019

Published Date: March 07, 2019

\begin{abstract}
For many years, we have been using a simplified reconstruction technique for scaphoid non-unions that involves the use of precise iliac bone graft first described by modified Matti-Russe technique, based on consideration of the three elements of bone healing, deep knowledge of the applied anatomy, vascularity and biomechanics of the carpal bone. We have retrospectively reviewed the results of 69 consecutive patients with nonunion of different sites of the scaphoid treated by one senior author between 2005 and 2018. The objectives of our technique are to apply simple step by step technique that can be utilize by an average orthopedic and hand surgeon starting from simple careful volar soft tissue dissection not to add insult to vasculature, simple reduction, excision of the fibrous none union part of the fracture, preparation and micro fractures of the fracture bed, precise bone graft harvesting, simple fixation and closure. This technique is simple, fast and precise, only used for an established scaphoid nonunion without significant arthritis, chronic non-union with bone cyst formation and may be used in recurrent non-union after failed surgery associated with DISI carpal deformity but no significant radiocarpal or mid carpal arthritis.
\end{abstract}

\section{Discussion}

McLaughlin 1954 "An unsolved problem"

Barton 1996"Awkward but important little bone"

Little has changed since 1950 still" Awkward but important little bone"

Green Etal "If all bones heal by the same process, why are scaphoid difficult to heal"

1. Failure of timely diagnosis

2. Systemic and local factors

3. Inappropriate treatment.

Bone healing is a complex process requiring mainly on

1. Viable bone cells

2. Continuous bone to bone contact with minimal shears at fracture site

\section{Blood supply}

Unfortunately the Scaphoid nonunion are lacking all the three elements for union
1. Bindra using microcomputer tomography found "bone trabeculae are more tightly packed and thick at the proximal pole and distal pole In contrast to the waist with the trabeculae are thinnest and sparsely with High numbers of fracture waist and the necessity for a supplement of viable bone cells with highest quality of bone graft.

2. Vascular anatomy: Gleeman in 1980 majority via radial artery $70-80 \%$ interosseous vascularity and entire proximal pole from dorsal vessels enter distally and dorsally travel proximally along the dorsal ridge, $20-30 \%$ volar radial artery arise from either the artery directly or the superficial palmar branch to enter through the tubercle region, so we do not dissect or violate the dorsal aspect of fracture site and preservation of the superficial palmar branch of the radial artery

3. In a Comparison of Two Percutaneous Volar Approaches for Screw Fixation of Scaphoid Waist Fractures, Radiographic and Biomechanical Study of an Osteotomy-Simulated Model [1] Figure 1 to 3 .

Conclusions: The data suggest that, in a cadaveric osteotomysimulated scaphoid waist fracture model, the transtrapezial 
approach reliably achieves central positioning of a screw in the proximal and distal poles. This position offers a biomechanical advantage compared with central placement in only the proximal pole, so we use simple $\mathrm{K}$ wires fixation and stopped using the screws fixation principle as it is bulky and needs violation of the scaphotrapezial joint with subsequent osteoarthritis Figure 1 to 3.

\section{Our principles}

1. Volar approach with no dorsal dissection and try to preserve the palmar branch of the radial artery because of the vascular supply pattern

2. Using precise iliac crest cortico cancellous bone graft as the best replaced bone cells supplement as well as bone marrow aspiration and small bone graft supplementation.

3. We use simple central K wire and don't use complex screw fixation with its occasional malposition, it is bulky and needs violating the STT joint with its subsequent osteoarthritis.

\section{Step by Step Technique}

Step 1 skin incision - volar approach over the FCR, curved at the styloid process level Figue 1, Minimal soft tissue dissection tries not to ligate the palmar branch of the radial artery, no posteriorlateral dissection hindering the vascularity, marking the capsule and ligaments for latter closure by stay vicryle sutures, special radiocarpal ligament approach

Step 2 Reduction and maintain the length by hyper extension of the wrist with ulnar deviation,

Special technique for Dorsal intercalated segment instability (DISI) by hyper flextion of the wrist and fixing the lunate bone in flexion position by driving K-Wire through the lower end of the radius and lunate, then hyper extension of the wrist and then proceed" [2]

Step 3 Punch out the two fragments of the fracture including fibrous nonunion or interposed tissue, don't go deep with the punch to the posterior cortex, leave it intact, first by manual prying then by very light hammering

Step 4 Prepare the bed to receive the graft with micro fracture till bleeding using small $1 \mathrm{~mm} \mathrm{k}$ wire and small curette, drive 1 or 2 $\mathrm{K}$ wires $1.2 \mathrm{~mm}$ or $1.4 \mathrm{~mm}$ ante or retrograde under vision and mark its maximum length in reduced position of the fracture under vision by marker then withdrew it to free the bone graft bed for later on driving it back to its pre marked length fixing the graft in situ [3-6]

Step 5 Via small skin incision over Ipsi lateral iliac crest and using the same punch size harvest a cortico cancellous wedge bone graft and prepare it to fit the fracture bed (remove the attached soft tissue), aspirate few 2-3 cc of bone marrow aspiration by a syringe and take few cancellous bone grafts as well [7]

Step 6 Insert the small bone grafts and impact them in fracture bed sides closing the small gaps in distal and proximal fragments then insert the main graft in its bed with the stronger bone cortex facing volar and cancellous bone inside to maintain the volar length and reduction, then push the $\mathrm{k}$ wire for fixing the graft till the full marked pre-determined length, inject the bone marrow aspirate, no need for $\mathrm{x}$ ray control .

Step 7 No wash out, closure the ligament, the capsule and the skin, injection of Marcaine 5 cc .5\% sub cuticular. POP cast in slight flexion and radial deviation to relax the ligaments for 6 weeks [810]

Post-operative: Removal of the pop cast in 2 weeks for stiches removal and change pop cast for 4 weeks more or otherwise better to keep the pop cast for the 6 weeks if you use absorbable sutures and the cast in good condition.

Start physiotherapy later after removal of the pop cast and the fixing k wires in the clinic after 6 -8 weeks postoperative [11]
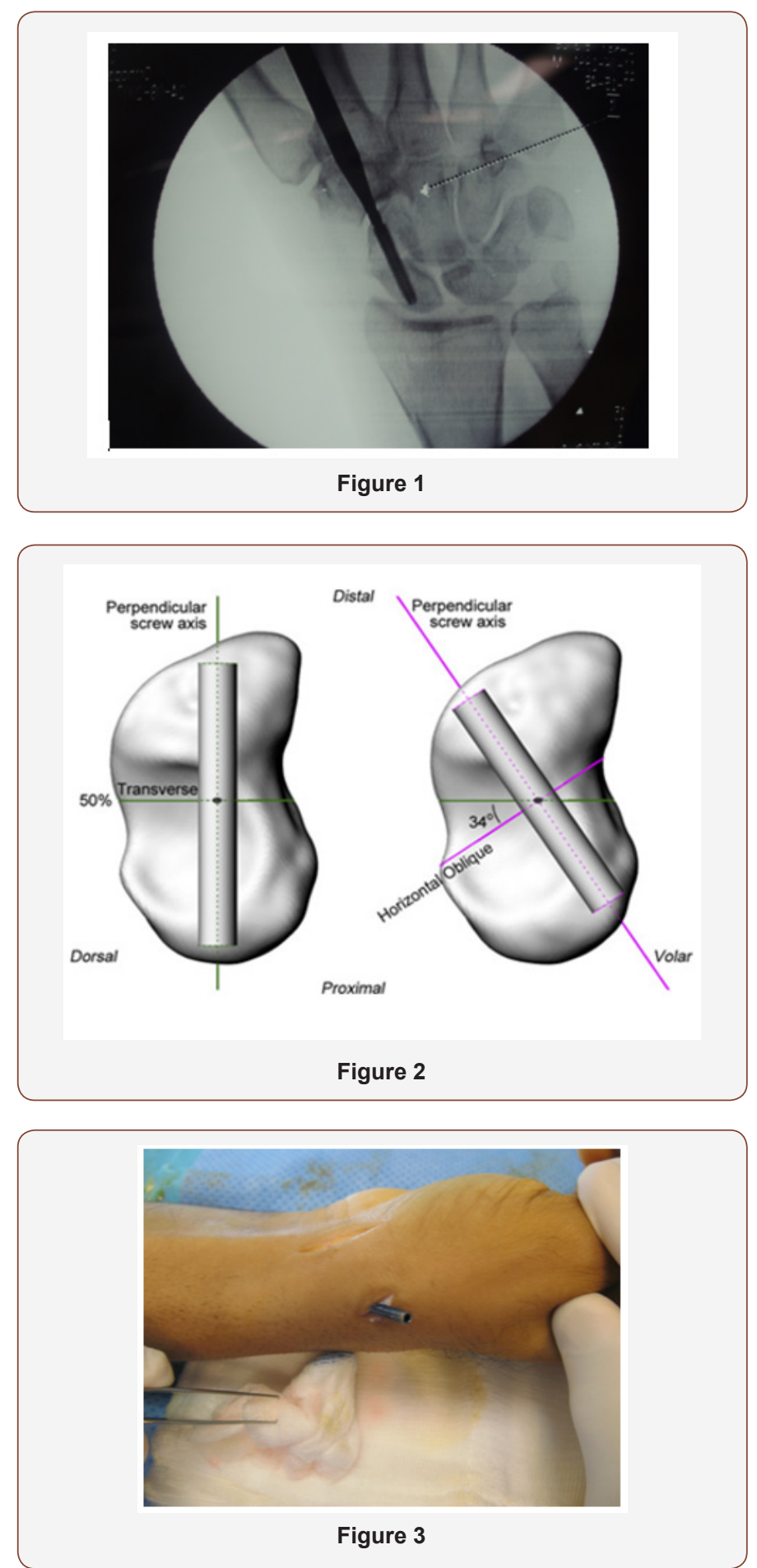

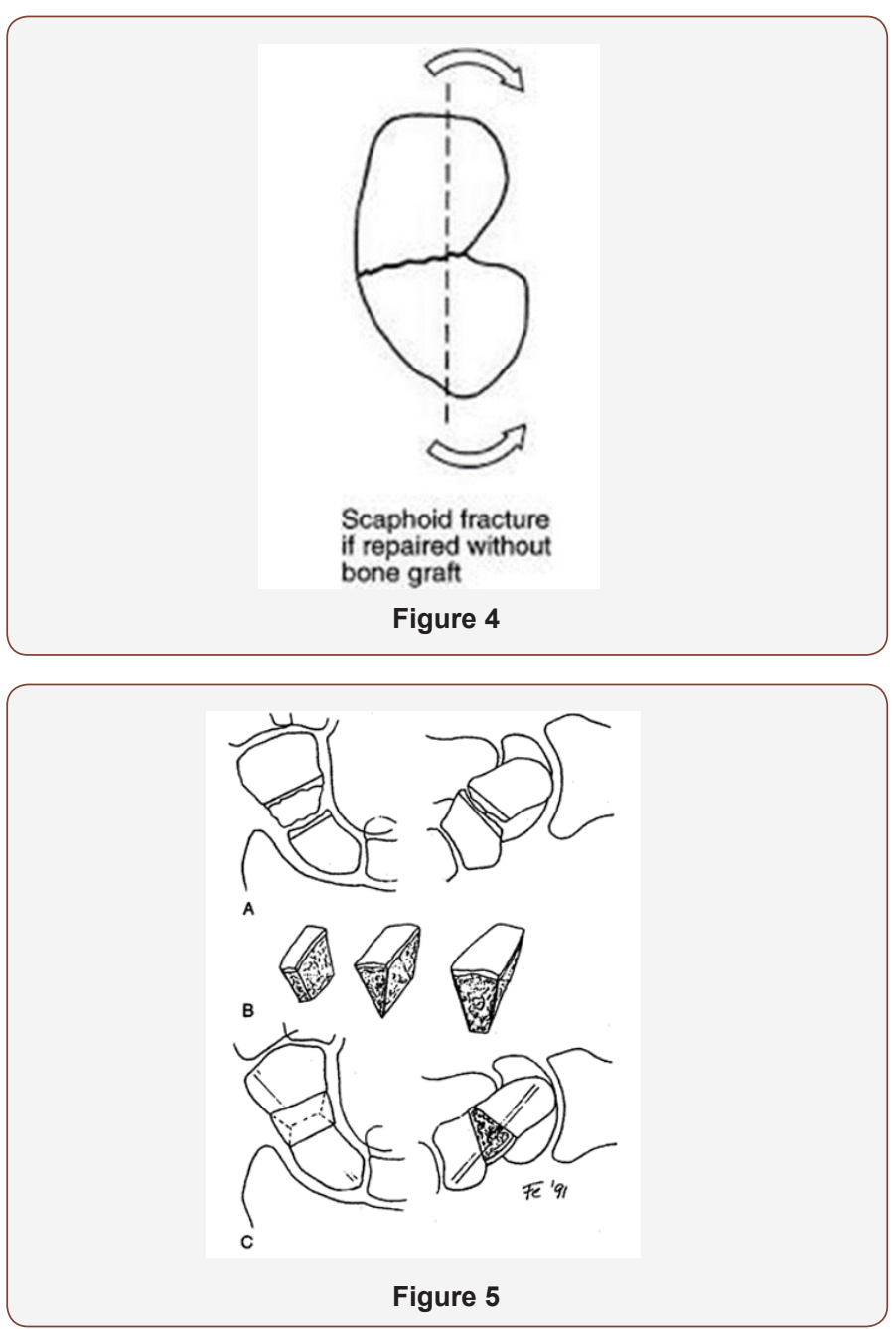

\section{Step 1}

Skin incision - volar approach over the FCR, curved at the styloid process level, Minimal soft tissue dissection try not to ligate the palmar branch of the radial artery, no posterior-lateral dissection hindering the vascularity, marking the capsule and ligaments for latter closure by stay vicryle sutures, special radiocarpal ligament approach.

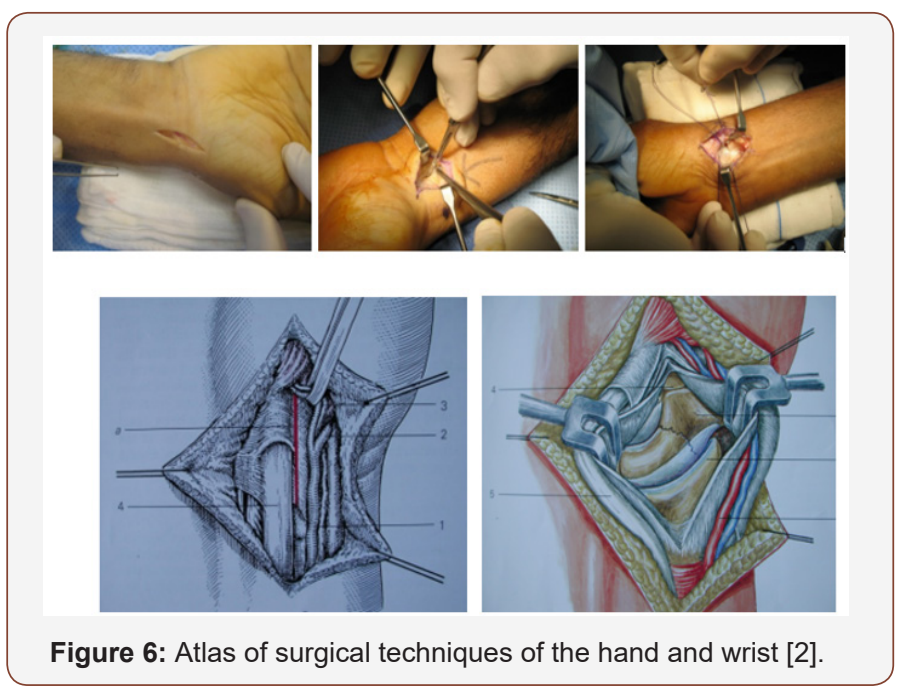

\section{Step 2}

Reduction and maintain the length by hyper extension of the wrist with ulnar deviation, Special technique for Dorsal intercalated segment instability (DISI) by hyper flextion of the wrist and fixing the lunate bone in flextion position by driving K-Wire through the lower end of the radius and lunate, then hyper extension of the wrist and then proceed.

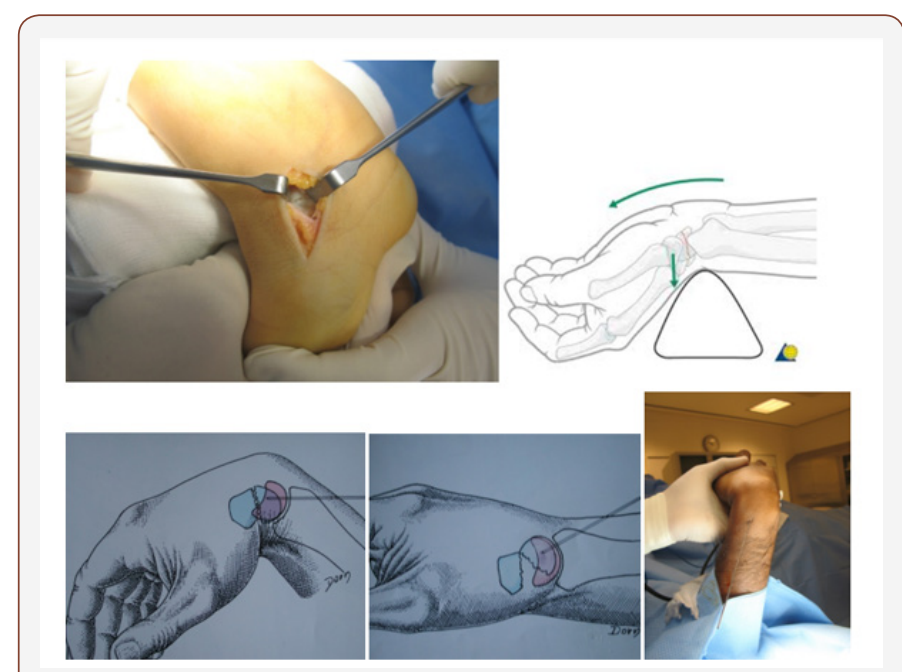

Figure 7: Atlas of surgical techniques of the hand and wrist [2].

\section{Step 3}

Punch out the two fragments of the fracture, including fibrous nonunion or interposed tissue, don't go deep with the punch to the posterior cortex, leave it intact, first by manual prying then by very light hammering.

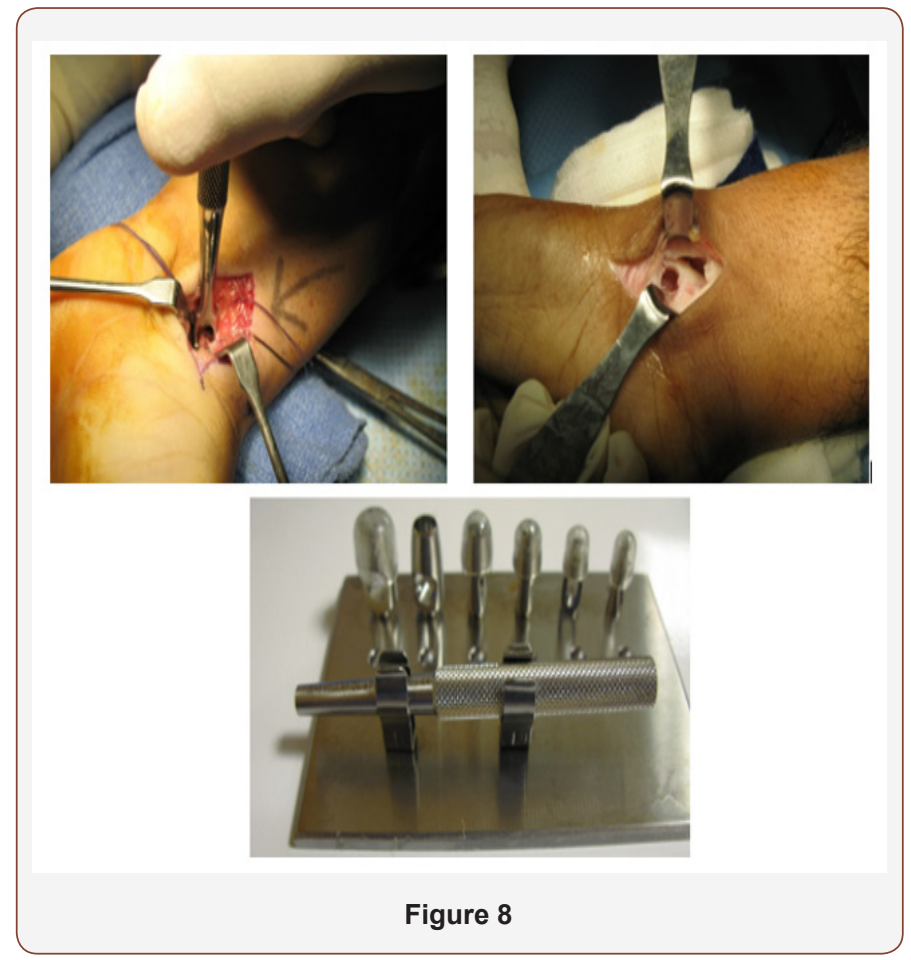

\section{Step 4}

Prepare the bed to receive the graft with micro fracture till bleeding using small $1 \mathrm{~mm} \mathrm{k}$ wire and small curette, drive 1 or $2 \mathrm{~K}$ wires $1.2 \mathrm{~mm}$ or $1.4 \mathrm{~mm}$ ante or retrograde under vision and mark its maximum length in reduced position of the fracture under vision by marker then withdrew it to free the bone graft bed for later on driving it back to its pre marked length fixing the graft in situ. 


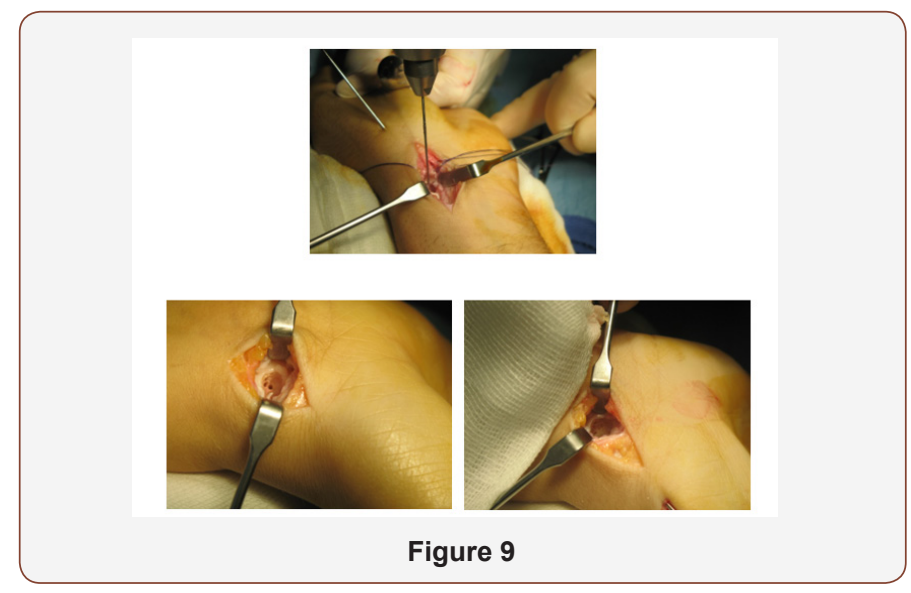

\section{Step 5}

Via small skin incision over Ipsi lateral iliac crest and using the same punch size, harvest a corticocancellous wedge bone graft and prepare it to fit the fracture bed (remove the attached soft tissue), aspirate few 2-3 cc of bone marrow aspiration by a syringe and take few cancellous bone grafts as well.

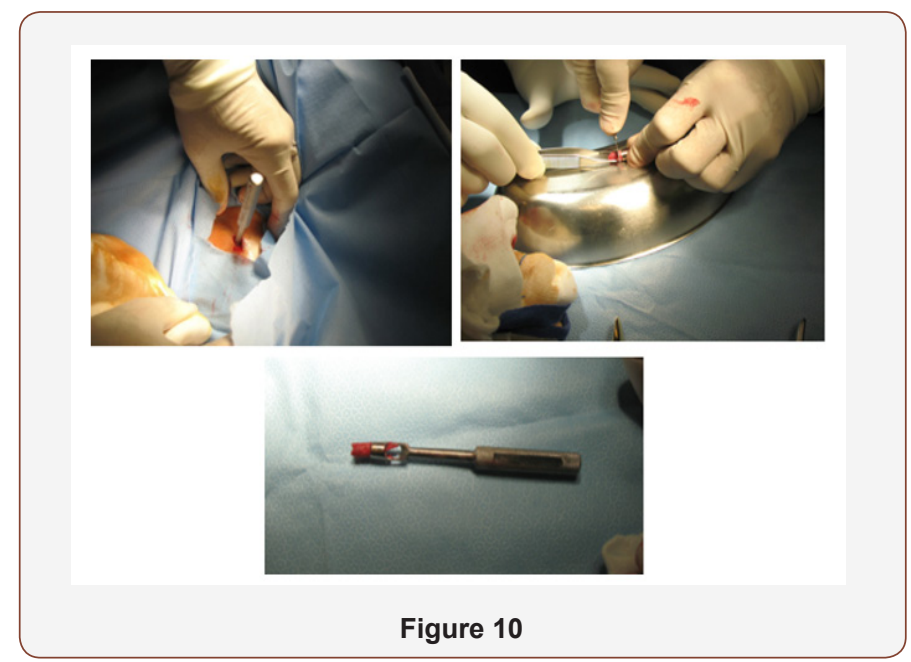

\section{Step 6}

Insert the small bone grafts and impact them in fracture bed sides closing the small gaps in distal and proximal fragments then insert the main graft in its bed with the stronger bone cortex facing volar and cancellous bone inside to maintain the volar length and reduction, then push the $\mathrm{k}$ wire for fixing the graft till the full marked pre-determined length, inject the bone marrow aspirate, no need for $\mathrm{x}$ ray control.

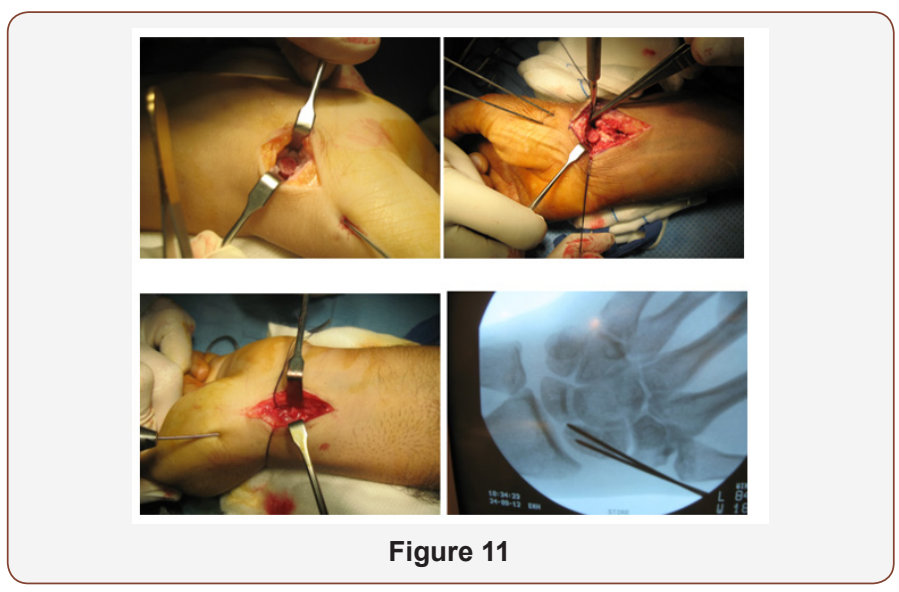

\section{Step 7}

No wash out, Closure of the ligament and capsule supported sometime with FCR sheath, skin closure, injection of Marcaine $5 \mathrm{cc}$ $.5 \%$ sub cuticular. POP cast in slight flexion and radial deviation to relax the radiocarpal ligaments for 6 weeks.

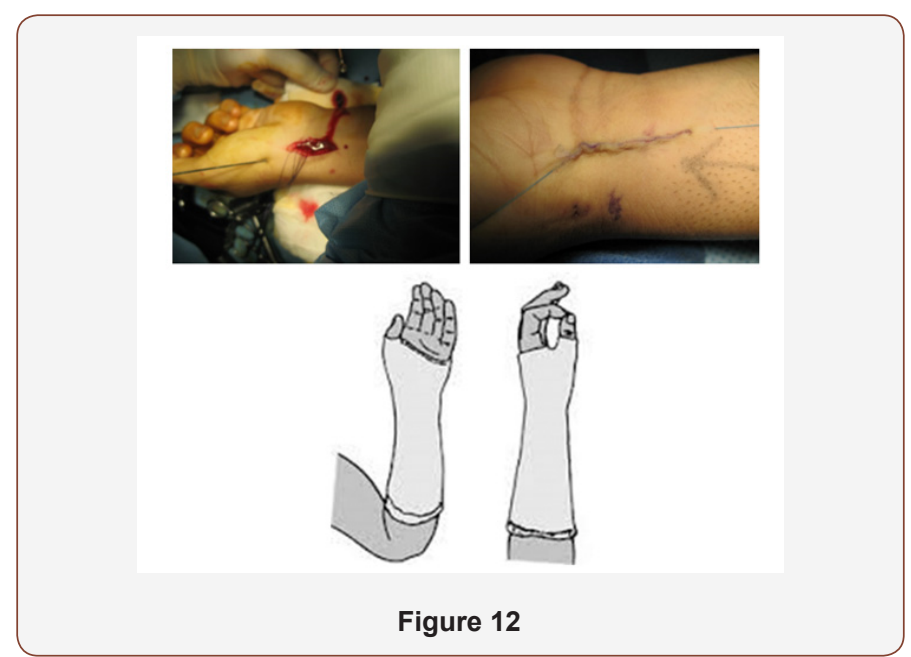

\section{Post-operative}

Removal of the pop cast in 2 weeks for stiches removal and change pop cast for 4 weeks more or otherwise better to keep the pop cast for the 6 weeks if you use absorbable sutures and the cast in good condition, Start physiotherapy later after removal of the pop cast and the fixing $\mathrm{k}$ wires in the clinic after $6-8$ weeks postoperative.

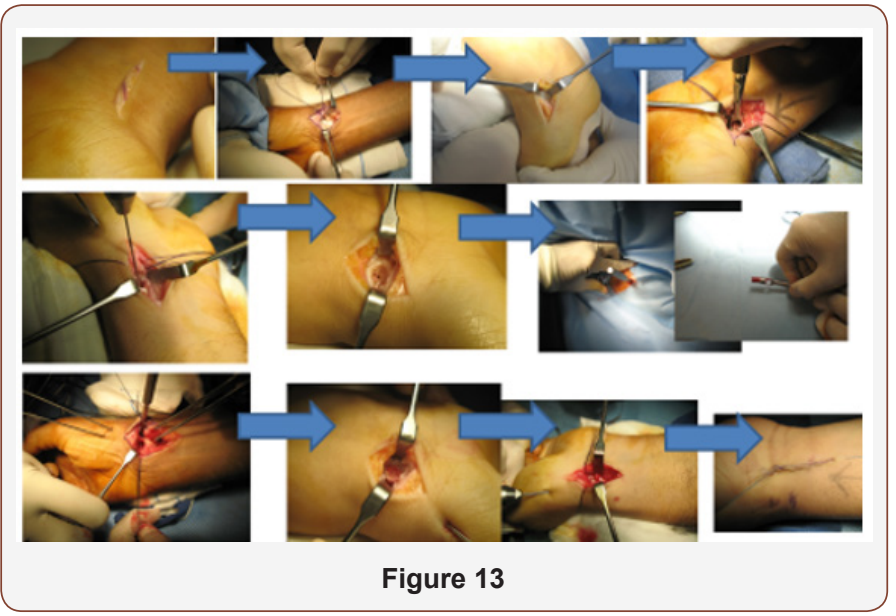

Acknowledgement

None.

\section{Conflict of Interest}

No Conflict of Interest.

\section{References}

1. Geert Meermans, Francis Van Glabbeek, Marc Braem, Roger van Riet, Guy Hubens, et al. (2014) Comparison of Two Percutaneous Volar Approaches for Screw Fixation of Scaphoid Waist Fractures: Radiographic and Biomechanical Study of an Osteotomy-Simulated Model. The Journal of Bone and Joint Surgery 96(16): 1369-1376.

2. Raoul tubiana, Alan Gilbert, Alain C Masquelet (1999) Atlas of surgical techniques of the hand and wrist.

3. Scott Wolfe, William Pederson, Scott H Kozin (2010) Green's Operative Hand Surgery. Churchill Livingstone $6^{\text {th }}$ Edition 2: 1-2392. 
4. Evenski AJ, Adamczyk MJ, Steiner RP, Morscher MA, Riley PM (2009) Clinically suspected scaphoid fractures in children. J Pediatr Orthop 29(4): 352-355.

5. Pierre Jerome C, Moncayo V, Albastaki U, Terk MR (2009) Multiple occult wrist bone injuries and joint effusions: prevalence and distribution on MRI. Emerg Radiol 17(3): 179-184.

6. Ramos Escalona J, García Bordes L, Martínez Galarza P, Yunta Gallo A (2010) Ulnar variance and scaphoid fracture. J Hand Surg Eur 35(3):195197.

7. Ramamurthy C, Cutler L, Nuttall D, Trail IA, Stanley JK, et al. (2007) The factors affecting outcome after non-vascular bone grafting and internal fixation for nonunion of the scaphoid. J Bone Joint Surg Br 89(5): 627632.
8. Mayfield JK, Gilula LA, Totty WG (1992) Isolated carpal fractures. In: Bralow L (ed.) The Traumatized Hand and Wrist: Radiographic and Anatomic Correlation. Philadelphia, Pa: WB Saunders, USA, pp. 249-263.

9. Metz VM, Gilula LA (1993) Imaging techniques for distal radius fractures and related injuries. Orthop Clin North Am 24(2): 217-228.

10. Rogers LF (1992) The wrist. In: Radiology of Skeletal Trauma. New York, NY: Churchill Livingstone $2^{\text {nd }}$ edn, USA, pp. 837-938.

11. Dias JJ, Finlay DB, Brenkel IJ, Gregg PJ (1987) Radiographic assessment of soft tissue signs in clinically suspected scaphoid fractures: the incidence of false negative and false positive results. J Orthop Trauma 1(3): 205-208. 